Original article

\title{
Factors affecting blood donation intervals and patterns of return based on a sample in southern Iran: A follow-up design
}

\author{
Salman Daneshi ${ }^{1}$, Esmat Rezabeigi Davarani ${ }^{2}$, Farhad Arefi ${ }^{1}$, Fateme Jahangiri Mehr ${ }^{3}$, Kiavash Hushmandi ${ }^{4}$, \\ Mehdi Raei $^{5}$, Reza Fariabi ${ }^{1}$, Mohadeseh Shojaei Shahrokhabadi ${ }^{6}$ \\ 1 Jiroft University of Medical Sciences, Jiroft, Iran \\ 2 Kerman University of Medical Sciences, Kerman, Iran \\ ${ }^{3}$ Ahvaz Jundishapur University of Medical Sciences, Ahvaz, Iran \\ ${ }^{4}$ University of Tehran, Tehran, Iran \\ ${ }^{5}$ Baqiyatallah University of Medical Sciences, Tehran, Iran \\ ${ }^{6}$ University of Pretoria, Pretoria, South of Africa
}

Received 21 September 2020, Revised 11 May 2021, Accepted 26 July 2021

(C) 2020, Russian Open Medical Journal

\begin{abstract}
Objectives - The main goal of blood transfusion centers is to recruit and retain more qualified blood donors. This study aimed to identify the factors influencing the return of volunteer blood donors.

Material and Methods - A cohort study was conducted on all blood donors who donated blood for the first time, from March 21, 2013, to March 20, 2014. They were followed up for five years (up to March 20, 2018). Demographic characteristics, type of blood donation and time interval between two blood donations were recorded as recurrent events. Data were then analyzed using Logit Model for Multinomial Responses and Cox's shared frailty model.

Results - Of the 2010 blood donors, $33.7 \%$ as the repeated donor and $16.2 \%$ as the regular donor had regular donations. The frequency of blood donation was in the range of 1 to 20 with a mean of $4.54 \pm 3.29$. There was a significant reverse relationship between the number of donations and the time interval between two donations. The variables of gender, marital status and occupation had a significant effect on the blood donation of the repeated and regular donors. Genders, age at the time of the first donation, marital status and occupation had a significant effect on the intervals between blood donations.

Conclusions - Most of blood supply of Iran is provided by men and first-time donors. Since the decision to donate and keep donating blood involves many complex issues applied programs are require to convert women and first-time donors into regular donors.
\end{abstract}

Keywords: donor, recurrent event, transfusion, logit model.

Cite as Daneshi S, Davarani ER, Arefi F, Mehr FJ, Hushmandi K, Raei M, Fariabi R, Shahrokhabadi MS. Factors affecting blood donation intervals and patterns of return based on a sample in southern Iran: A follow-up design. Russian Open Medical Journal 2021; 10 : e0406.

Correspondence to Fateme Jahangiri Mehr. Phone: +989303383224, Email: Afsjm.90@gmail.com.

\section{Introduction}

Blood donation centers need to provide an adequate, healthy supply of blood [1]. To provide an adequate and healthy supply of blood products, it is beneficial to increase the number and rate of blood donation [2]. In Iran, most of blood donations are collected from non-remunerated and voluntary blood donors who can donate healthy blood which is a real asset to blood transfusion centers. repeated donations will reduce the errors resulting from unhealthy blood transfusion to a minimum $[3,4]$.

One of the most important goals of blood transfusion centers is to get healthy blood that in Iran is collected from voluntary blood donors who can donate healthy blood, which is a real asset to blood transfusion centers [3].

There are several type of blood donors in Iran, repeated donors who donate blood at least twice a year and regular donors who have previous experience of blood donation and first-time donors [5].
Repeated donors play an essential role in providing adequate, healthy blood supplies [6] Few donors are regular donors, an important question for those in charge of blood services which some of the people why go to these centers more often and especially willing to become regular donors. The time interval between two donations is an important factor in the donors' return and the number of donations $[4,6,7]$. Several studies have investigated the time interval between donations, however there is still insufficient data for factors affecting blood donation intervals [3, 8-10]. Moreover, there is scant data about how frequently these donors return to donate and, whether certain donation characteristics correlate with donor return.

Knowing the factors that affect blood donation will lead to regular donors. According to the standard of Iranian Blood Transfusion Organization, a regular donor donates blood, at least, twice a year and thus examined regularly with given screening tests that increase the chances of healthy blood transfusion 
regarding infectious diseases will reduce the errors resulting from unhealthy blood transfusion to a minimum [4]. The time interval between two donations is an important factor associated with repeated blood donation [7]. James and Matthews [8] studied the time interval between donation using survival regression in Canada and Ownby et al [7] studied the statistical basis of a return to donation using Cox model in the United States. They studied age, gender, and race and education level. In a study by Flegel et al. [9], the donor's chance of donating in a predictable time interval was estimated based on the logistic regression model. The results showed that the chance of donation depended on factors, such as the donor's age, gender, the frequency of donation, blood type and location of life.

In the same line, Kasraian and Tavassoli studied the relationship between blood donation and demographic characteristics in a 3-year period and the return rate for subsequent donation [10].

In another study in 2014, Kheiri et al. showed that age, sex, body weight, job class and the first-time donors, the frequency of donation during the first year played an important role in donor return behavior [3].

The objectives of this research are to identify the factors that affect patterns of blood donation and return to donation, frequency of donation and time interval between donations in the first-time donors as well as the factors that influence donors to continue or stop donating between March 21, 2013 until March 20, 2018 based on a sample in southern Iran with the use of regression models.

\section{Material and Methods}

This 5-year follow-up study was conducted in a blood transfusion center in southeast Iran (blood center of Kerman province). The study population included all volunteer blood donors who referred to the Blood Transfusion Center of Kerman for the first time, since 21 March 2013 to 20 March 2014, and whose age range was between 20 and 60 years. They were then followed up to 20 March 2018. The records of all blood donors, such as the number of redundant donations and the time interval between each donation, were received from the blood transfusion organization in Kerman province. Furthermore, demographic information, including age at the time of the first donation, gender, marital status, education level and occupation were collected based on the checklist through telephone call with blood donors. Typical inclusion criteria included demographic, clinical, and geographic characteristics. Blood donors were divided into three groups: 1. regular donor who have previous experience of blood donation, 2. repeated donor who donates blood at least twice a year, and 3. First-time donor. New and repeated donor status was assigned using the first donation during the calendar year.

This study was conducted according to the Declaration of Helsinki, and the ethics committee of kerman University of Medical Sciences, Iran, approved the study protocol. Written informed consent was obtained from all participants before they took part in the study.

\section{Statistical analysis}

The data were analyzed using the R-version 3.3.2 (R- Project, 2016) software. Logit Model for Multinomial Responses was used to examine the factors affecting the type of blood donation. Multinomial logistic regression is used to model nominal outcome variables, in which the log odds of the outcomes are modeled as a linear combination of the predictor variables. In this model, the type of blood donation (regular donor, repeated donor and firsttime donor) was considered as the response variable. Parametric estimations and standard error of the two Logit models were shown using the "first-time" category as the basic ca tegory [11].

In order to analyze the factors affecting blood donation intervals, blood donation was considered as a "recurrent event" and the time interval between two blood donations was considered as a "recurrent survival time". For donors who did not re-donate, the recorded time was defined as the censored survival time. Furthermore, the interval between the last donation and the end of the study was recorded as censored survival data. The donation times (time lags) for each individual are dependent on unknown factors. Statistical models methods can be used to deal with unknown factors. Therefore, in order to eliminate the effect of this dependence on the estimation of regression parameters, the Cox's shared frailty model (extension of Cox proportional hazard model) was used [12].

Table1. The frequency of the type of blood donation and its association with demographic characteristics

\begin{tabular}{|c|c|c|c|c|c|}
\hline \multirow{2}{*}{ Variable } & \multirow{2}{*}{ Category } & \multicolumn{3}{|c|}{ Types of blood donation } & \multirow{2}{*}{$p$-value } \\
\hline & & First-time & Repeated & Regular & \\
\hline \multirow{2}{*}{ Sex } & Male, 1812 (90.1) & $857(47.3)$ & $640(35.3)$ & $315(17.4)$ & \multirow{2}{*}{$<0.001$} \\
\hline & Female, 198 (9.9) & $150(75.8)$ & $38(19.2)$ & $10(5.0)$ & \\
\hline \multirow{2}{*}{ Marital status } & Single, $465(23.1)$ & $309(66.5)$ & $112(24.0)$ & $44(9.5)$ & \multirow{2}{*}{$<0.001$} \\
\hline & Married, 1545 (76.9) & $698(45.2)$ & $566(36.6)$ & $281(18.2)$ & \\
\hline \multirow{5}{*}{ Employment status } & Unemployed, 400 (19.9) & $287(71.8)$ & $96(24.0)$ & $17(4.2)$ & \multirow{5}{*}{$<0.001$} \\
\hline & Office Clerk, 655 (32.7) & $277(42.3)$ & $248(37.9)$ & $130(19.8)$ & \\
\hline & Worker, 30 (1.4) & $12(40.0)$ & $13(43.3)$ & $5(16.7)$ & \\
\hline & Self-employed, 861 (42.8) & $400(46.5)$ & $303(35.2)$ & $158(18.3)$ & \\
\hline & Retired, 64 (3.2) & $31(48.4)$ & $18(28.2)$ & $15(23.4)$ & \\
\hline \multirow{4}{*}{ Educational level } & Illiterate, 79 (4.0) & $44(53.2)$ & $288(33.6)$ & $113(13.2)$ & \multirow{4}{*}{$<0.001$} \\
\hline & Primary, 398 (19.8) & 195 (49.0) & $137(34.4)$ & $66(16.6)$ & \\
\hline & Secondary, 857 (24.6) & $456(53.2)$ & $288(33.6)$ & $113(13.2)$ & \\
\hline & University, 676 (33.6) & $312(46.2)$ & $230(34.0)$ & $134(19.8)$ & \\
\hline Total $(\mathrm{N}=2010)$ & & $1007(50.1)$ & $678(33.7)$ & $325(16.2)$ & \\
\hline
\end{tabular}

Data are presented as frequencies (\%). 
Table2. Results of multi-category Logit regression model for factors associated with type of blood donation

\begin{tabular}{lccccc}
\hline Type of donation & Variable & Coefficient & SE & $p$-value & OR \\
\hline \multirow{3}{*}{ Repeated } & Intercept & -1.906 & 0.604 & 0.001 & - \\
& Gender (male) & 0.826 & 0.223 & 0.0002 & 2.285 \\
- & Marital status (single) & -0.621 & 0.155 & $<0.001$ & 0.537 \\
& Intercept & -1.903 & 0.769 & 0.013 & - \\
Regular & Gender (male) & 0.751 & 0.370 & 0.043 & 2.119 \\
& Marital status (Single) & -0.540 & 0.209 & 0.009 & 0.583 \\
& Employment status & -1.337 & 0.471 & 0.004 & 0.263 \\
\hline
\end{tabular}

Basic category: first-time donation.

Table 3. Results of the Cox's shared frailty models for analyzing time between donations

\begin{tabular}{|c|c|c|c|}
\hline Variable & Coefficient & $S E$ & $p$-value \\
\hline \multicolumn{4}{|l|}{ Donor duration } \\
\hline Second vs. first & -0.023 & 0.04 & 0.556 \\
\hline Third vs. first & -0.045 & 0.043 & 0.298 \\
\hline Fourth vs. first & -0.032 & 0.046 & 0.482 \\
\hline Fifth vs. first & -0.094 & 0.049 & 0.054 \\
\hline Sixth vs. first & -0.209 & 0.053 & $<0.001$ \\
\hline Seventh vs. first & -0.149 & 0.058 & 0.01 \\
\hline Eight vs. first & -0.268 & 0.064 & $<0.001$ \\
\hline Ninth vs. first & -0.22 & 0.07 & 0.001 \\
\hline Tenth vs. first & -0.219 & 0.081 & 0.007 \\
\hline Eleventh vs. first & -0.032 & 0.09 & 0.725 \\
\hline Twelfth vs. first & -0.059 & 0.104 & 0.568 \\
\hline Thirteenth vs. first & -0.379 & 0.126 & 0.003 \\
\hline Fourteenth vs. first & -0.475 & 0.159 & 0.002 \\
\hline Fifteenth vs. first & -0.463 & 0.211 & 0.028 \\
\hline Sixteenth vs. first & -0.867 & 0.332 & 0.009 \\
\hline Seventeenth vs. first & -0.424 & 0.414 & 0.306 \\
\hline Eighteenth vs. first & 0.184 & 0.503 & 0.714 \\
\hline Nineteenth vs. first & 0.007 & 0.743 & 0.993 \\
\hline Twentieth vs. first & -0.751 & 3.858 & 0.846 \\
\hline Gender (male) & 0.297 & 0.102 & 0.004 \\
\hline Age & -0.022 & 0.003 & $<0.001$ \\
\hline Marital status (single) & -0.497 & 0.076 & $<0.001$ \\
\hline \multicolumn{4}{|l|}{ Employment status } \\
\hline Clerk vs. retired & -0.366 & 0.132 & 0.005 \\
\hline Self-employed vs. retired & -0.437 & 0.131 & 0.001 \\
\hline Unemployed vs. retired & -0.994 & 0.143 & $<0.001$ \\
\hline Random effect & - & - & $<0.001$ \\
\hline
\end{tabular}

\section{Results}

In this study, 1812 (90.1\%) of the 2010 blood donors were males. The mean age \pm SD for the volunteers was $34.0 \pm 11.0$ years. The age range of the volunteers was between 18 and 65 years.

The number of blood donations during the 5 years was 1 to 20 with a mean \pm SD of $4.54 \pm 3.29$ and a total of 10674 times. The frequency of the type of blood donation and its association with demographic characteristics are shown in Table 1.

The mean time interval between the first and second donations, the second and third donations, the third and fourth donations, the fourth and fifth donations, and with a decreasing trend, between nineteenth and twentieth donations was respectively $351 \pm 339,293 \pm 258,244 \pm 193,221 \pm 157$, and $91 \pm 2$ days. Spearman's correlation coefficient shows a significant reverse relationship between the number of donations and the time interval between two donations (Spearman's rho $=-0.36, p$ value<0.001). In other words, those who have the highest number of blood donations have come for a shorter time interval for donation.
The results of the multinomial Logit regression model are presented in Table 2. Based on the results of this model, gender and marital status had a significant effect on blood donation of the repeated donors. The estimated Odds Ratio of "repeated donors" vs. "first-time donors" for men is 2,285 times as much for women. Additionally, the estimated OR corresponding to marital status $(O R=0.537)$ can be interpreted as: "being single decreases the chance of being "repeated donors" vs. "first-time donors" by $46.3 \%$.

Furthermore, the variables of gender, marital status and unemployed occupational group vs. retired donors had a significant effect on the donation of regular blood donors. The estimated odds ratio of "regular donors" vs. "one-time donors" for men is 2.119 times as much for women.

The estimated OR corresponding to marital status ( $O R=0.583$ ) can be interpreted as: "being single decreases the chance of being "repeated donors" vs. "first-time donors" by $41.7 \%$. Also, in terms of job the estimated OR (0.263) can be interpreted as: "being unemployed decreases the chance of being "repeated donors" vs. "first-time donors" by $73.7 \%$.

The results of the Cox proportional hazard model, despite the effect of fragility for recurrent events of blood donation for simultaneous analysis of all recurrent survival times, have been recorded in Table 3. According to the results, the intervals between the second to the fifth, the eleventh and the twelfth and the seventeenth to the twentieth, with the time interval to the first visit, had no significant difference. There was a significant difference between the first-time intervals with the sixth to the tenth and the thirteenth to the sixteenth time intervals. Similarly, gender, age at the time of the first donation, marital status and occupation group showed a significant effect on time intervals between blood donations. Men had more chances of blood donation than women. In other words, more men have referred for blood transfusion. At the time of the first donation, age has a negative effect on the time interval between blood donations, i.e., volunteers with ageless have come for a shorter time interval for donation and have a higher chance of re-donating. Single donors have had a lower chance of having blood donations than married donors; in other words, married people have come to donate less often at intervals. Volunteers who are the clerk, self-employed, non- employed have had little chance re-donation than retired donors, meaning they are less likely to come to re-donation compared to a retired career group.

\section{Discussion}

The most interesting finding in our study was that the blood donation rate for first-time donors, repeated donors and regular donors was $50.1 \%, 33.7 \%$ and $16.2 \%$ respectively. The findings of the current study differ from Farshadpour's study who showed that the majority of donors were regular donor followed by first time donors and repeated donors [13]. It is difficult to explain this difference, but it might be related to factors, such as sample size, period and place of study.

The re-donation rate in our study was $49.9 \%$. The findings of the current study are consistent with those of Kasraian and Kheiri who found the re-donation rate $51.7 \%$ and $49.1 \%$, respectively [10]. The re-donation rate in China and Brazil was $36 \%$ and $61.1 \%$ respectively [14]. This result may be explained by the fact that the average proportion of the population donating in developing countries is almost similar. 
The results of this study also showed a statistically significant negative association between donation rate and the time interval in two blood donations. It seems that increasing the number of donations, decreases the interval between two donations. This finding is in agreement with Kheiri and Alibeigi's findings [3].

Another important finding of this study was the statistically significant association observed between the time intervals. However, the dependence between time intervals is one of the main features of time intervals between blood donations which involve many complex issues such as Personal characteristics, sense of altruism, social responsibility and knowledge about donation.

In the current study also showed a statistically significant negative association between age and time intervals. It seems that the younger people have the shorter interval between two donations compared to old people. Although, these results differ from few published studies [7]. they are consistent with the findings of other previous study reporting higher re- donation for the younger people [3].

The results of this study also showed the participation of male and female donors was $90.1 \%$ and $9.9 \%$ respectively. In addition, the results of two models showed a statistically significant association between gender and time intervals between two blood donations. It seems that male donors were more likely to return than female donors. This finding supports previous research which indicated the low contribution of women to blood donation in Iran [15]. However, the findings of the current study do not support the previous research in other countries [14-17]. Therefore, low participation of women in Iran, requires increasing women's awareness of the importance of blood donation and identifying the factors leading to women's less blood donation as well as planning country-specific programs to address these barriers [15].

In the present study it was shown that, $66.5 \%$ of first-time donors, $24.1 \%$ of repeated donors, and $9.5 \%$ of regular donors were single. In addition, the results of multi-category Logit regression model showed a statistically significant association between marital status and type of blood donation. Moreover, the results of Cox regression model showed a statistically significant association between marital status and time intervals between two blood donations. It seems that married donors were more likely to return than single donors. This finding is in agreement with Kasraian and Tavassoli's findings which showed that the significant correlation between marital status and blood donation in the second and third years. However, scant research has been conducted on the association between marital status and blood donation. A possible reason for this, can be the high correlation between age and marital status [10].

In the current study, the highest and the lowest rates of blood donation belonged to self-employed and the working class, respectively. Our findings also showed that occupation had an effect on the time interval between two blood donations. This also accords with our earlier observations, which showed that job had an effect on return to donation $[3,14]$. Similarly, Guo et al. showed that employed people had a higher donation than students [14].

In the present study, $45.3 \%$ of first-time donors and $42.5 \%$ of repeated donors held a diploma. Additionally, $41.2 \%$ of regular donors had university education. review of the literature indicates that donors with higher education levels are more likely to become regular blood donors in developing countries [6, 14]. These results differ from Guo's finding which showed that people with lower education had more blood donations than people with higher education. A Possible explanation for this, is the free use of blood for their relatives. In fact, the level of education is directly related to the occupation and people with lower education levels have less income-generating jobs [14]. The results of this study did not show any association between level of education and frequency of donation and time intervals between two blood donations. The findings of the current study are consistent with those of Kheiri, Kasraian and Tavassoli who did not find any correlation between blood donation rate the level of education of the donors [10].

\section{Conclusion}

In general, the findings of this study indicate that gender, age, marital status and occupation of donors have a significant effect on the time interval between blood donations; therefore, the reduction of the time interval between two blood donations and the first- time and last- time blood donors converting to regular donors needs planning and targeting at blood transfusion centers. One of these plans can be the appropriate behavior with donors and their recall for blood re-donation. The results of studies conducted in Iran show that first-time donors are likely to become regular donors if they are invited to receive donations from invitations or telephone calls for re-donating, 3 to 4 months after the first blood donation.

On the other hand, in addition to maintaining regular donors, as the most important source of healthy blood supply, the supply and consumption of blood and blood products is an important principle in community health management.

\section{Limitations}

We acknowledge some limitations of our study. The sample size was small. Therefore, the results of the study may not be simply generalized to the whole population. Moreover, period of study was short and this can cause changes in the results.

\section{Conflict of interest}

The authors have no conflict of interest to declare.

\section{Acknowledgment}

The authors gratefully acknowledge the Department of Transfusion of Kerman Province for data. The authors would like to sincerely thank the participants of the study and especially their colleagues in the Department of Transfusion of Kerman Province who helped to recruit, interview, and examine the study participants.

\section{Ethical approva}

All procedures performed in studies involving human participants were in accordance with the ethical standards of Kerman University of Medical Sciences committee and with the 1964 Declaration of Helsinki and its later amendments or comparable ethical standards.

\section{References}

1. Sojka BN, Sojka P. The blood donation experience: self-reported motives and obstacles for donating blood. Vox Sang 2008; 94(1): 56 63. https://doi.org/10.1111/j.1423-0410.2007.00990.x. 
2. Kasraian L. Causes of discontinuity of blood donation among donors in Shiraz, Iran: cross-sectional study. Sao Paulo Med J 2010; 128(5): 272 275. https://doi.org/10.1590/S1516-31802010000500006.

3. Kheiri S, Alibeigi Z. An analysis of first-time blood donors return behaviour using regression models. Transfus Med 2015; 25(4): 243248. https://doi.org/10.1111/tme.12177.

4. Schreiber GB, Sanchez AM, Glynn SA, Wright DJ. Increasing blood availability by changing donation patterns. Transfusion 2003; 43(5) :591-597. https://doi.org/10.1046/j.1537-2995.2003.00388.x.

5. Gharehbaghian A, Abolghasemi H, Namini MT. Status of blood transfusion services in Iran. Asian J Transfus Sci 2008; 2(1): 13-17. https://doi.org/10.4103/0973-6247.39505.

6. Schreiber GB, Sharma U, Wright D, Glynn S, Ownby H, Tu Y, et al. First year donation patterns predict long-term commitment for first-time donors. Vox Sang 2005; 88(2): 114-121. https://doi.org/10.1111/j.1423-0410.2005.00593.x.

7. Ownby H, Kong F, Watanabe K, Tu Y, Nass CC. Analysis of donor return behavior. Retrovirus Epidemiology Donor Study. Transfusion 1999; 39(10): 1128-1135. $\quad$ https://doi.org/10.1046/j.15372995.1999.39101128.x.

8. James R, Matthews D. Analysis of blood donor return behaviour using survival regression methods. Transfus Med 1996; 6(1): 21-30. https://doi.org/10.1046/j.1365-3148.1996.d01-46.x.

9. Flegel WA, Besenfelder W, Wagner FF. Predicting a donor's likelihood of donating within a preselected time interval. Transfus Med 2000; 10(3): 181-192. https://doi.org/10.1046/j.1365-3148.2000.00251.x.

10. Kasraian L, Tavassoli A. Relationship between first-year blood donation, return rate for subsequent donation and demographic characteristics. Blood Transfus 2012; 10(4): 448-452. https://doi.org/10.2450/2012.0097-11.

11. Agresti A. An introduction to categorical data analysis. 2nd ed. Hoboken, New Jersey: John Wiley \& Sons, Inc. 2007; 372 p. https://mregresion.files.wordpress.com/2012/08/agresti-introductionto-categorical-data.pdf.

12. Cook RJ, Lawless J. The statistical analysis of recurrent event. New York: Springer. 2007; 404 p. https://doi.org/10.1007/978-0-387-69810$\underline{6}$.

13. Farshadpour F, Taherkhani R, Tajbakhsh S, Gholizadeh Tangestani M, Hajiani G, et al. Prevalence and trends of transfusion-Transmissible viral infections among blood donors in south of Iran: An Eleven-Year retrospective study. PloS One 2016; 11(6): e0157615. https://doi.org/10.1371/journal.pone.0157615

14. Guo N, Wang J, Yu Q, Yang T, Dong X, Wen G, et al. Long-term return behavior of Chinese whole blood donors. Transfusion 2013; 53(9): 1985-1991. https://doi.org/10.1111/trf.12142.

15. Al Shaer L, Sharma R, AbdulRahman M. Analysis of blood donor predonation deferral in Dubai: characteristics and reasons. J Blood Med 2017; 8: 55-60. https://doi.org/10.2147/jbm.s135191.

16. Marantidou O, Loukopoulou L, Zervou E, Martinis G, Egglezou A Fountouli $P$, et al. Factors that motivate and hinder blood donation in Greece. Transfus Med 2007; 17(6): 443-450. https://doi.org/10.1111/j.1365-3148.2007.00797.x.

17. Wiersum-Osselton JC, Marijt-van der Kreek T, Brand A, Veldhuizen I, van der Bom JG, de Kort W. Risk factors for complications in donors at first and repeat whole blood donation: a cohort study with assessment of the impact on donor return. Blood Transfus 2014; 12 Suppl 1 (Suppl 1): s28-36. https://doi.org/10.2450/2013.0262-12.

\section{Authors:}

Salman Daneshi - PhD Candidate of Epidemiology, instructor, Department of Public Health, School of Health, Jiroft University of Medical Sciences, Jiroft, Iran. https://orcid.org/0000-0002-9199-0382.

Esmat Rezabeigi Davarani - PhD Candidate, Research Center for Health Services Management, Institute of Futures Studies in Health, Kerman
University of Medical Sciences, Kerman, Iran. https://orcid.org/0000-00032129-3562.

Farhad Arefi - PhD Candidate, Instructor of Medical Surgical Nursing, Department of Nursing, School of Nursing, Jiroft University of Medical Sciences, Jiroft, Iran. https://orcid.org/0000-0002-3423-1646.

Fateme Jahangiri Mehr - MSc of Biostatistics, Lecturer, Biostatistics and Epidemiology Department, Ahvaz Jundishapur University of Medical Sciences, Ahvaz, Iran. https://orcid.org/0000-0001-5742-4531.

Kiavash Hushmandi - PhD Candidate, Department of Food Hygiene and Quality Control, Faculty of Veterinary Medicine, University of Tehran, Tehran, Iran. https://orcid.org/0000-0001-5682-5392.

Mehdi Raei - PhD of Biostatistics, Assistant Professor of Biostatistics, Health Research Center, Life Style Institute, Baqiyatallah University of Medical Sciences, Tehran, Iran. https://orcid.org/0000-0002-5899-4744.

Reza Fariabi - PhD of Health Education \& Promotion, Assistant Professor of Health Education \& Promotion, Department of Public Health, School of Public Health, Jiroft University of Medical Sciences, Jiroft, Iran. https://orcid.org/0000-0003-3753-7031.

Mohadeseh Shojaei Shahrokhabadi - PhD of Biostatistics, Assistant Professor of Biostatistics, University of Pretoria, Pretoria, South of Africa. https://orcid.org/0000-0003-0623-8565. 